\title{
Color Image Enhancement with Different Image Segmentation Techniques
}

\author{
Rupali B. Nirgude \\ D.Y. Patil Institute of Engineering and Technology \\ Ambi, Pune
}

\begin{abstract}
Image can be improved using different image enhancement techniques. It includes different types of operations like image segmentation, clustering, smoothing, etc. Basically in the process of Image segmentation features having homogenous characteristics are identified. The Input for the system is color image. The image gets converted into horizontal and vertical shape histogram. Then cluster formation is done using hill climbing technique and $\mathrm{k}$ means clustering. $\mathrm{K}$ Means clustering consider colour intensity as criteria. Sequential probability ratio test checks similar characteristics between different regions. Merging of these regions follows dynamic region merging algorithm. Depending on similar properties partitions are merged. The output is enhanced segmented image. Nearest neighbour graph technique is helpful to speed up the above process. This improved image is useful in the field of medical as well as security purpose.
\end{abstract}

\section{Keywords}

NNG, DRM, SPRT

\section{INTRODUCTION}

Image segmentation is nothing but to divide the image into number of pieces[1] that have similar features, group the meaningful pieces for the convenience of perceiving. Segmentation helps to modify the image into many regions which are basically larger representation of data, where region is group of connected pixels with similar properties. The goal of segmentation is to simplify the representation of an image into something that is more meaningful and easier to analyze. Image segmentation is typically used to locate objects and boundaries in images.

In this paper plane color image is the input. The image is made of various colors. Considering various features of image, it gets converted into regions using hill climbing technique. Here color clusters are formed using $\mathrm{K}$ means clustering segmentation techniques, the last step is to recover the image. This is possible using Sequential probability ratio test, nearest neighbour and dynamic region merging algorithm here SPRT test assume some predicate to check the consistency. Once consistency is checked then nearest neighbor graph and dynamic region merging algorithm is used for better segmented output image. With the help of these algorithm the neighbouring regions are progressively merge. DRM algorithm gives automatic segmentation.

There are different image segmentation methods[2] like Kmeans (KM) [8] Clustering Methods, Histogram-Based Methods, Edge Detection Methods, Region Growing Methods, Model based, set level Methods, Graph Partitioning Methods, Watershed Transformation, Neural Networks Segmentation, and Multi-scale Segmentation. Relay Level set method [9], [10].

\section{LITERATURE REVIEW}

In the field of image segmentation huge research is done in last some years. Image segmentation is divide the image into subparts which are divided according to certain standard, Image segmentation is having wide variety of methods. Some of the methods discuss in detail as follows

A) Threshold segmentation: It is famous technique of image segmentation as it is easy to use. This divide the input image mainly into two exact different parts where one with segment value as positive and second is with the segment value as negative. First some dimension for the image regions are set, and then segment all regions according to the dimension. It can also use horizontal and vertical Histogram techniques to partition the image. [11]

B) Edge segmentation: In this method the operation starts from outer region to inner region. First identify the edges of the image. Then process these outer edges so that only boundaries of the regions is visible, with this identify the object with this technique considering edge as criteria. It is generally used to identify these objects. [3]- [5]

C) Region based segmentation: Here similarity between neighboring regions is observed. If neibhouring regions are maximum similar then merge these region. Likewise proceed further up to stop criteria. [12]- [15]

D) Clustering techniques: clustering is one of most famous techniques in segmentation. Clustering is nothing but group of similar type of elements. K-means algorithm is famous example of clustering. Clusters are formed by calculating the centroid and objects near to centroid. Clustering is mainly divided into two types top down approach and bottom up approach [7] [8]

E) Matching: In this method set the ideal object, and search for other object which having same feature as that of the ideal object. Locating the objects is possible with this type. This type of segmentation is known as matching.

\section{Overview of the system}

- Color image is input for this system. Color clusters are formed using $\mathrm{k}$ means clustering method. Auto clustering approach is helpful here. The plane color image is converted into horizontal and vertical histogram.

- Then we apply hill climbing technique to detect the peak points, in short peak detection is possible using histogram and hill climbing technique.

- Then form the actual clusters with the help of Kmeans clustering algorithm. Here clusters are made of various regions.

- Check similarity between these regions. Consistency of the region is calculated by sequential probability ratio test, which depend on the two fix 
assumptions. Right assumption and wrong assumption. Then sort the data into two different groups.

- $\quad$ Finally apply principal of nearest neighbor graph. It will allow better merging at the initial stage. Then use dynamic region merging which gives automatic image segmentation.at this step actual merging is possible, Output of the DRM algorithm is enhanced segmented image.

\section{FLOW OF SYSTEM}

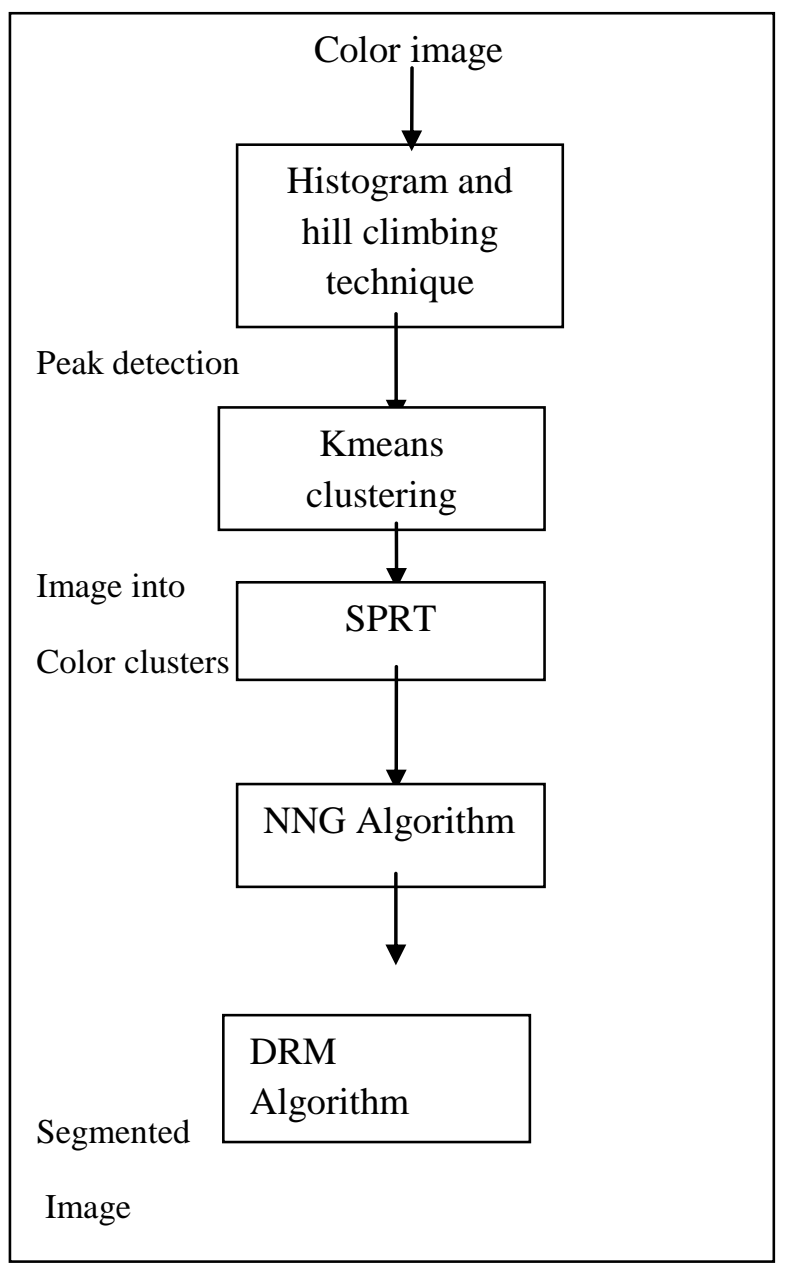

Fig 1.Flow of System

\section{HISTOGRAM AND HILL CLIMBING TECHNIQUE}

This is the technique which is applied to plane color image color image is converted into histogram. There are two approaches of histogram techniques, horizontal histogram and vertical histogram. With the help of hill climbing technique histogram of the color image helps to detect the peaks. These peaks are number of clusters which are formed from color image.

\section{K MEANS CLUSTERING}

$\mathrm{K}$ means clustering popular techniques used to form the color clusters. As mention above once the peaks in hill climbing process are detected then actual clustering starts. Here number of peaks will be equal to number of clusters.

The $\mathrm{K}$ means clustering algorithm works as follows Construct a partition of $\mathrm{n}$ documents into a set of $\mathrm{K}$ clusters.
- Input: An image and the number K.

- Output: A partition of $\mathrm{K}$ clusters where K IS number of peaks.

- $\quad$ Select $\mathrm{K}$ random docs $\{\mathrm{S} 1, \mathrm{~S} 2, \ldots . . \mathrm{SK}\}$ as peaks

- Form Clusters based on centroids and its distance to neighboring objects.

- Reassign clusters, which are based on distance to the current cluster centroids.

- This process is continuing up to stopping criteria.

\section{CONSISTENCY TEST(SPRT)}

This test checks homogenous features in the image. Consistency property deals with two conditions: true condition and false condition. The condition in which similarity of the adjacent region is considered, it is known as true condition and condition considering the dissimilarity is known as the false condition. For true condition combine the regions. This is explaining as follows:

Here consider two assumptions to check if the regions are homogenous or not.

- Result=true, if neighboring regions are similar in desired features, then we merge the regions easily. It is also called as valid hypothesis.

- Result=false, if neighboring regions are not similar .or very contradictory features are present then hypothesis is known as alternative hypotheses.

The algorithm for consistency test is as below

- $\quad$ Set $\lambda_{1}$

- Choose $\lambda_{2}=1, \alpha=0.05, \beta=0.05$

- $\mathrm{A}=\log (1-\beta / \alpha), \mathrm{B}=\log \beta(1-\alpha)$

- Choose the $\mathrm{k}$ pixels of neighboring regions.

- The predicate cue $\mathrm{x}$ is calculated which require $\mathrm{P} 0\left(\mathrm{x} / \theta_{0}\right), \mathrm{P} 1\left(\mathrm{x} / \theta_{1}\right)$

- This value is calculated as

$\mathrm{P}_{0}\left(\mathrm{x} \mid \theta_{0}\right)=\lambda_{1} \exp \left(-\left(\mathrm{I}_{\mathrm{b}}-\mathrm{I}_{\mathrm{a}+\mathrm{b}}\right)^{\mathrm{T}} \mathrm{S}_{\mathrm{I}}^{-1}\left(\mathrm{I}_{\mathrm{b}}-\mathrm{I}_{\mathrm{a}+\mathrm{b}}\right)\right)$

$\mathrm{P}_{1}\left(\mathrm{x} \mid \theta_{1}\right)=1-\lambda_{2} \exp \left(-\left(\mathrm{I}_{\mathrm{b}}-\mathrm{I}_{\mathrm{a}}\right)^{\mathrm{T}} \mathrm{S}_{\mathrm{I}}^{-1}\left(\mathrm{I}_{\mathrm{b}}-\mathrm{I}_{\mathrm{a}}\right)\right)$

- Calculate $\delta=\delta+\log \left(\mathrm{P}_{0}\left(\mathrm{x} \mid \theta_{0}\right) /\left(\mathrm{P}_{1}\left(\mathrm{x} \mid \theta_{1}\right)\right.\right.$

If $\delta>=\mathrm{A}$, then regions are consistent.

If $\delta>=\mathrm{B}$, then regions are not consistent

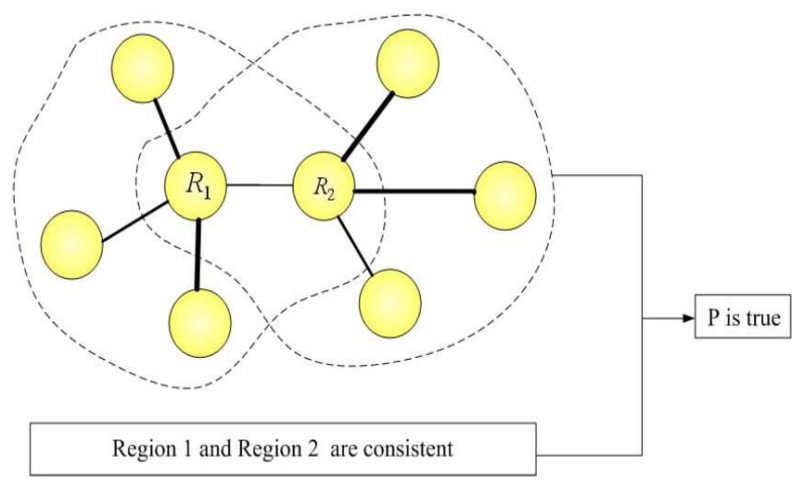

Fig 2.Consistency test 


\section{NEAREST NEIGHBOR GRAPH}

Once the consistency of the regions is check, start grouping the color clusters. With the help of NNG scanning very small part of image is possible, so it becomes very fast and efficient [17].

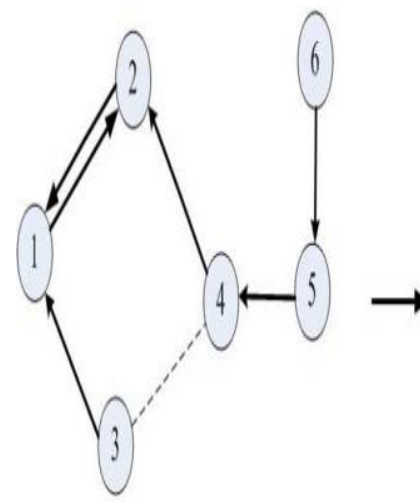

(a)

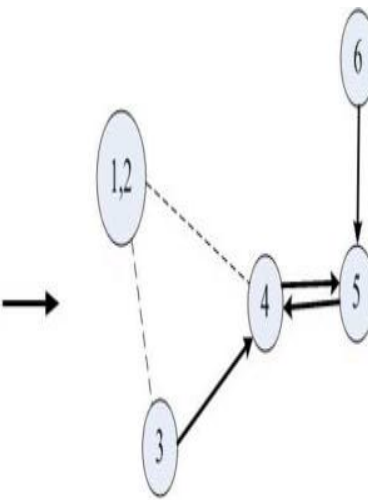

(b)
Fig 3.NNG Process

- Most similar pair of regions is connected by an edge with the minimum weight.

$$
\begin{aligned}
& \text { Consider } \\
& \text { dissimilarity=maximum similarity }
\end{aligned}
$$

\section{DYNAMIC REGION MERGING ALGORITHM}

There are different algorithms to merge the data. Though NNG is used at the initial stage, DRM is use at final step to merge the data because with the help of DRM we get better, fine and automatic segmentation. A region predicate is compared with the description of adjacent region; if both are same; merge them into a larger region. Otherwise regions are mention as different regions. We cannot combine such regions. This process will be continuing for all the regions in the image. If any region cannot merge with any of its adjacent neighbor, then at that point we have to stop as all the possible regions are already merged. This algorithm is conducted like discrete system. The segmentation in this algorithm is not overflow and not underflow. It is optimum. The DRM algorithm works as follows:

- Start with input image and give each region separate name.

- Likewise we can consider such k names.

- Give first name as B0 and proceed like the same

- Give last name to last region as B k.

- Start with first name up to last name.

- Obtain optimal solution for combining the regions

- Optimum solution can be obtain by dynamic programming

- Depending on the above solution, calculate the similarity weight between neighbor regions

- Minimum dissimilarity =maximum similarity is the only criteria needs to follow. Likewise go on combining the regions up to stopping criteria
The DRM algorithm diagrammatic representation is as below:

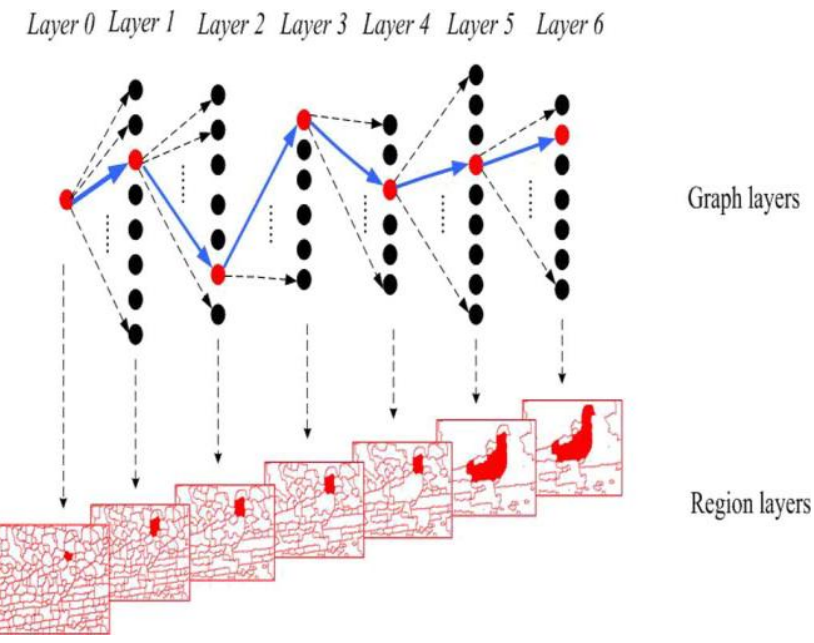

Fig 4.Dynamic region merging process

Dynamic region merging process works on the principal of the shortest path the corresponding image regions of each label layer. Starting from lower layer 0, (which is in red) we can obtain new label from (in red) its closest neighbor. If the region is merged with its neighbor, they will obtain to the same name of label.

DRM algorithm is very simple than rest of the merging algorithms. it gives better efficiency than any other algorithm. So it is better to use the DRM from efficiency point of view. The output of the DRM algorithm is segmented image. These output images are very useful as they give us detail and enlarge explanation of data; especially in military and medical applications. The alternative to the DRM algorithm are mean shift algorithm [16] as well as graph based algorithm [14]

\section{PROPOSED SOFTWARE DESIGN}

Interactive software is developed to do the reliable monitoring and management of segmentation process. The system software is made using MATLAB 10 .We are implementing hill climbing technique and $\mathrm{k}$ Means clustering first on the plane color image, and then applying consistency test using SPRT. Dynamic region merging algorithm and nearest neighbor graph on color image. This operation is totally software part. In the proposed DRM method, there are five parameters that control the consistency condition. While implementing the system there are four fix parameters, they are $\alpha, \beta, \lambda 1, \lambda 2$.Here $(\alpha, \beta)$ represent the probability of accepting an "inconsistent" model as "consistent" and rejecting a "consistent" model as "inconsistent" .m is used to decide the amount of data selected for the random test. If we set $\lambda 2=1$, then only $\lambda 1$ is the user input which can be vary. 


\section{RESULT}

Following images are used to represent results.

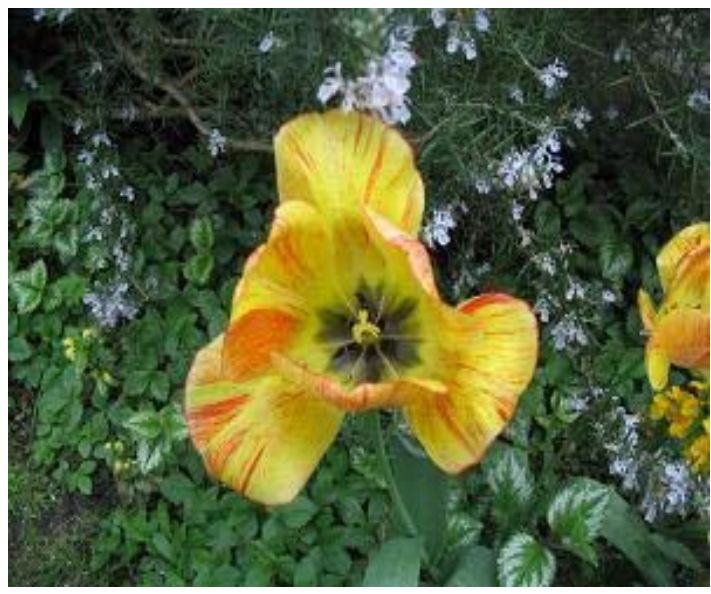

Input color image

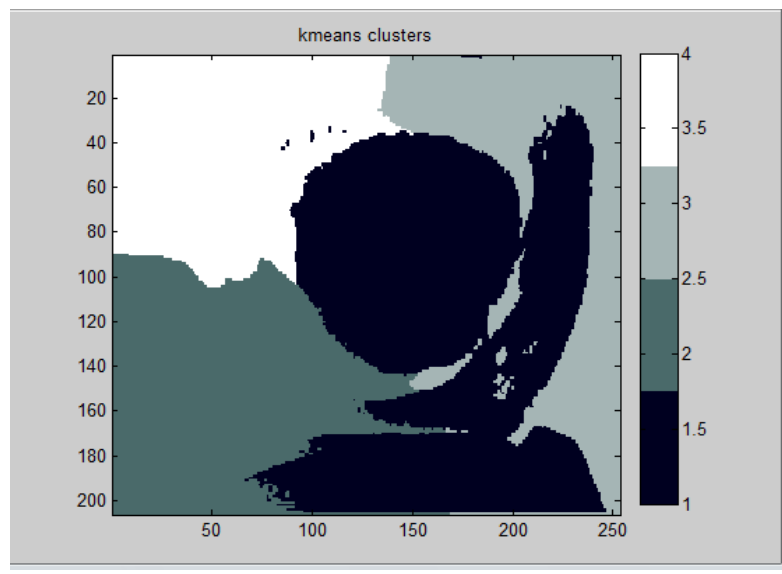

Region based segmentation

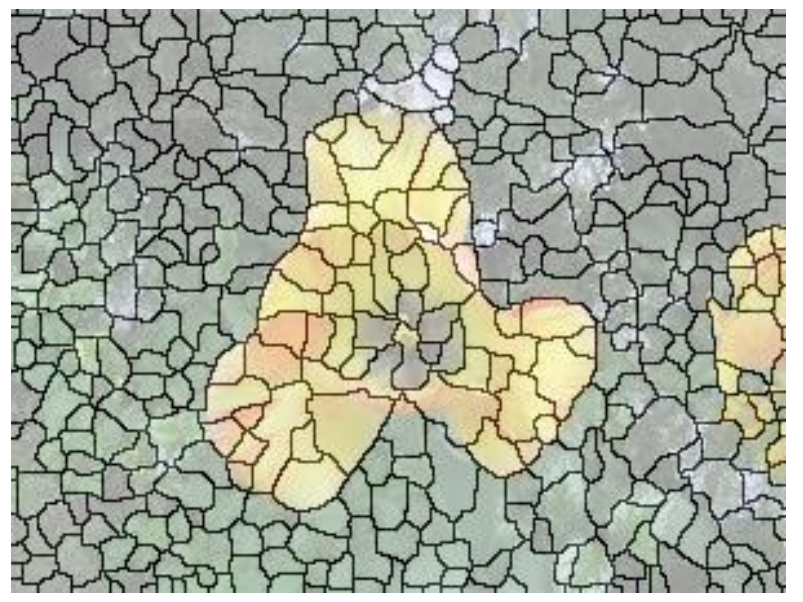

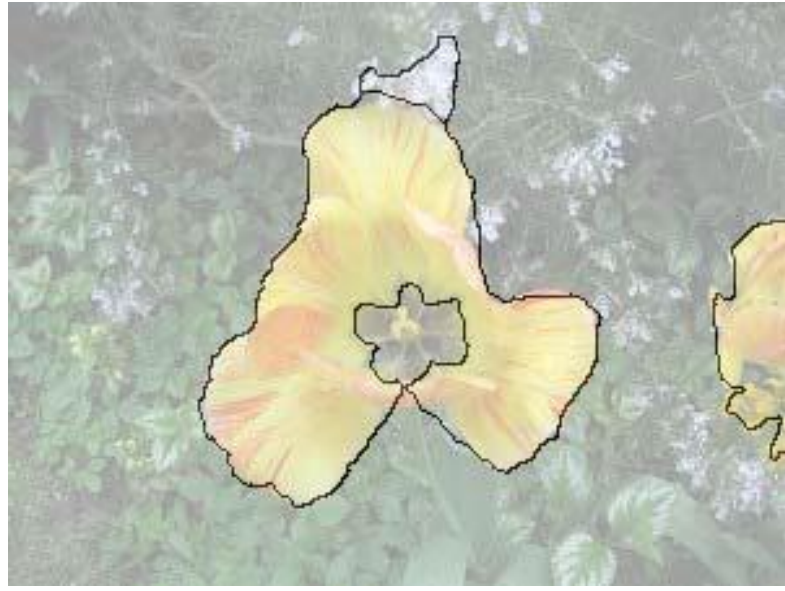

3) Output segmented image

\section{CONCLUSION}

Thus in this paper the proposed system suggest the image improvement technique with the help of different algorithms such as hill climbing technique ,K means clustering, consistency test, dynamic region merging algorithm, nearest neighbor graph at different stages. The system gives us knowledge about how to check the consistency of the regions according to the predefined criteria. These image improvement techniques have great future in medical and electronics. The extension to above mention paper is to increase the efficiency and also increase in tolerance. We can also increase the speed of the operation using advance algorithms.

\section{REFERENCES}

[1] D. A. Forsyth and J. Ponce, Computer Vision: A Modern Approach. Englewood Cliffs, NJ: Prentice-Hall, 2002.

[2] L. Ladicky, C. Russell, P. Kohli, and P. Torr, "Associative hierarchical CRFs for object class image segmentation," in Proc. ICCV, 2009, pp.

[3] J. Canny, "A computational approach to edge detection," IEEE Trans.Pattern Anal. Mach. Intell., vol. PAMI-8, no. 6, pp. 679-698, Nov.1986739-746.

[4] R. C. Gonzalez and R. Elwood, Digital Image Processing. Reading, MA: Addison-Wesley, 1992.

[5] L. Zhang and B. Paul, "Edge detection by scale multiplication in wavelet domain," Pattern Recognition. Lett., vol. 23, no. 14, pp.1771-1784, Dec. 2002.

[6] B. Paul, L. Zhang, and X.Wu, "Canny edge detection enhancement byscale multiplication," IEEE. Trans. Pattern Anal. Mach. Intell., vol. 27, no. 9, pp. 1485 1490, Sept. 2005

[7] Dorin Comaniciu, Peter Meer, "Mean Shift Analysis and Applications".

[8] S. Thilagamani1 and N. Shanthi, "A Survey on Image Segmentation Through Clustering", International Journal of Research and Reviews in Information Sciences Vol. 1, No.

[9] F. Lecumberry, A. Pardo, and G. Sapiro, "Simultaneous object classification and segmentation with high-order Wesley, 1992.multiple shape models", IEEE Trans. Image Process., vol. 19, no. 3, pp. 625-635, Mar. 2010. 
[10] R. C. Gonzalez and R. E. Woods, Digital Image Processing. Reading, MA: Addison

[11] National Programs on Technology Enhanced learning http://nptel.iitm.ac.in/courses/106105032/39,

[12] R. Nock and F. Nielsen, "Statistic region merging," IEEE Trans. PatternAnal. Mach. Intell., vol. 26, no. 11, pp. 1452-1458, Nov. 2004.

[13] K. Haris, S. N. Estradiadis, N. Maglaveras, and A. K. Katsaggelos, "Hybrid image segmentation using watersheds and fast regionmerging," IEEE Trans. Image Process., vol. 7, no. 12, pp. 1684-1699,Dec. 1998.

[14] P. F. Felzenszwalb and D. P. Huttenlocher, "Efficient graph-basedimage segmentation," Int. J. Computed. Vis., vol. 59, no. 2, pp. 167-181,Sep. 2004.
[15] B. Peng, L. Zhang, and J. Yang, "Iterated graph cuts for image segmentation, "in Proc. Asian Conf. computed. Vis., 2009, pp. 677-686

[16] D. Comanicu and P. Meer, "Mean shift: A robust approach toward featurespace analysis," IEEE Trans Pattern Anal. Mach. Intell., vol. 24, no. 5, pp. 603-619, May 2002.

[17] D. Eppstein, M. S. Paterson, and F. F. Yao, "On nearestneighborgraphs," Discr. Comput. Geom., vol. 17, no. 3 , pp. 263-282, Apr.1997.

[18] A. Wald, Sequential Analysis, 3rd ed. Hoboken, NJ: Wiley, 1947. 